\title{
Using Nonlinear Dynamics and Multivariate Statistics to Analyze EEG Signals of Insomniacs with the Intervention of Superficial Acupuncture
}

\author{
Shi-Yi Qi $\mathbb{D},{ }^{1}$ Dong Lin $\left(\mathbb{D},{ }^{1}\right.$ Li-Li Lin, ${ }^{1}$ Xiao-Zhen Huang $\mathbb{D},{ }^{2}$ Shen Lin $\mathbb{D}^{1},{ }^{1}$ Yun-Ying Yu, ${ }^{3}$ \\ Chuan-Hai Cao $\mathbb{1}^{4},{ }^{4}$ and Zhi-Xin Wang $\oplus^{5}$ \\ ${ }^{1}$ College of Acupuncture, Fujian University of Traditional Chinese Medicine, Fuzhou, Fujian Province, China \\ ${ }^{2}$ Department of Traditional Chinese Medicine Rehabilitation, Anxi County Hospital, Quanzhou, Fujian Province, China \\ ${ }^{3}$ Department of Sleep Medicine, Rehabilitation Hospital Affiliated to Fujian University of Traditional Chinese Medicine, Fuzhou, \\ Fujian Province, China \\ ${ }^{4}$ Department of Neurosurgery and Brain Repair, Morsani College of Medicine, University of South Florida, Tampa, FL, USA \\ ${ }^{5}$ Department of Internal Medicine, Florida Orthopedic Institute, University of South Florida, Tampa, FL, USA
}

Correspondence should be addressed to Dong Lin; bluelike1@sina.com

Received 5 August 2020; Revised 20 October 2020; Accepted 22 October 2020; Published 17 November 2020

Academic Editor: Chan-Yen Kuo

Copyright (c) 2020 Shi-Yi Qi et al. This is an open access article distributed under the Creative Commons Attribution License, which permits unrestricted use, distribution, and reproduction in any medium, provided the original work is properly cited.

\begin{abstract}
Objective. As a noninvasive and nonpharmacological therapeutic approach, superficial acupuncture (SA) is a special method of acupuncture. In this study, using nonlinear dynamics and multivariate statistics, we studied the electroencephalography (EEG) of primary insomnia under SA intervention to investigate how brain regions change. Method. This study included 30 adults with primary insomnia. They underwent superficial acupuncture at the Shangen acupoint. The EEG signals were collected for 10 minutes at each state, including the resting state, the intervention state, and the postintervention state. The data were conducted using nonlinear dynamics (including approximate entropy (ApEn) and correlation dimension (CD)) and multivariate statistics. Result. The repeated-measures ANOVA results showed that both ApEn and CD values were not significantly different at the three states $(p>0.05)$. The paired $t$-test results showed that the ApEn values of electrodes O2 (the right occipital lobe) at the postintervention state have decreased, compared with the resting state $(p<0.05)$, and no difference was detected in $\mathrm{CD}(p>0.05)$. The cluster analysis results of ApEn showed that patients' EEG has changed from the right prefrontal lobe (electrode Fp2) to the right posterior temporal lobe (electrode T6) and finally to the right occipital lobe (electrode O2), before, during, and after the SA intervention. In addition, the factor analysis results of CD revealed that patients' EEG of all brain regions except for the occipital lobes has changed to the frontal lobes and anterior temporal and frontal lobes from pre- to postintervention. Conclusion. SA activated the corresponding brain regions and reduced the complexity of the brain involved. It is feasible to use nonlinear dynamics analysis and multivariate statistics to examine the effects of SA on the human brain.
\end{abstract}

\section{Introduction}

Insomnia is one of the most common sleep disorders and can have negative impacts on patients' quality of life; it is also associated with medical morbidity $[1,2]$. Approximately a third of the population experience at least a mild form of insomnia, with $6-10 \%$ even meeting the diagnostic criteria for insomnia syndrome [3, 4]. Unfortunately, current treatments often produce adverse effects, such as drug dependence, depression, and amnesia $[3,5]$. Therefore, it is necessary and crucial to explore nondrug treatments for insomnia [6].

Acupuncture is a treatment that has recently gained more and more attention from researchers as a nonpharmaceutical treatment for insomnia $[7,8]$. A number of studies showed that insomnia is associated with hyperarousal in daily life, as well as overactivation of the central nervous system (CNS) and the autonomic nervous system 
(ANS) [9, 10]. Studies have also demonstrated that acupuncture not only can improve many diseases of the CNS, such as Alzheimer's [11], focal ischemic stroke [12], etc. but also causes autonomic remodeling by improving the balance between the vagus nerve and the sympathetic nervous system $[13,14]$.

Superficial acupuncture (SA) (alias shallow acupuncture) is a special form of acupuncture that was first recorded in the Internal Classic of the Yellow Emperor (Huang Di Neijing) in ancient China. SA is noninvasive, as it is blunt and placed on the surface of the skin without piercing and penetrating into it (see Figure 1(b)). Nowadays, it is even smaller and more exquisite. The needle is about $9 \mathrm{~cm}$ in length, and the needle handle is about $6 \mathrm{~cm}$ in length, which is wound with copper wire wrapping around. The acupuncturist held the needle handle with two right-hand fingers (index finger and middle finger) and scraped the needle handle at a uniform velocity for many minutes with his or her thumb to generate vibration wave at the acupoint. SA is widely used in Fujian province, China, and an increasing number of acupuncturists practice SA therapy to treat various kinds of diseases, including insomnia and prosopoplegia. Moreover, clinical studies have found that insomniacs show more sensitivity to pinprick stimuli [15], so some noninvasive treatments like SA decrease tension and effectively improve sleep quality in chronic insomnia patients [16, 17]. Unfortunately, despite being an effective treatment for insomnia, the mechanism of SA is still unknown.

Recent studies showed that electroencephalograms (EEG) signals are time series and it could be used to assess changes in brain activity in real time [18] as EEG signals are highly complex, having nonlinear and nonstationary behavior [19]. Studies have also indicated that understanding brain dynamics in different time domains is through nonlinear dynamical analysis of EEG $[20,21]$, which is a branch of the emerging holistic science of complex systems [22]. In nonlinear dynamical systems, there is no relationship between independent and dependent variables, meaning that this system has the typical characteristics of coherence, inhomogeneity, and asymmetry [23]. Therefore, in this study, the Approximate Entropy (ApEn) and Correlation Dimension (CD) were used to extract the feature of EEG signals. With good antinoise and anti-interference abilities, the ApEn requires only a short data point to estimate the random signals and then determine the characteristics of the whole signal $[20,24]$, while CD is also considered one of several key measures in chaotic time series analysis, which can assess the degree of similarity in the time series between one observation and others [25].

Furthermore, the "complexity" of a living system, such as a human brain, is not a single uniform process or property that is easily captured with a single variable $[25,26]$. Using simple conventional statistical methods arbitrarily divides the whole brain into a number of independent components without taking into account the group structure problem among the brain regions. Thus, the statistical results and conclusions obtained from these studies cannot accurately describe the central effect of SA. Therefore, the use of a combination between nonlinear analysis and multivariate statistics EEG measures gave the advantages to investigate the changes in different brain regions over time.

The structure of this paper is as follows. In the "Methods" section, the clinical trial procedure of SA and the corresponding nonlinear analysis methods are introduced. In the "Results" section, the results and corresponding data analysis are presented. Finally, the "Discussion" section is given. Overall, in this study, using nonlinear dynamics and multivariate statistics, we studied the EEG of primary insomnia under SA stimulation to determine what the implications are on the brain. The results and the proposed method in our study will be useful in laying a foundation for further study on the mechanism through which SA affects brain function.

\section{Methods}

2.1. Design. Focused on these changes of brain regions strongly implicated in superficial acupuncture intervention, this study was designed to compare EEG signals before, during, and after SA stimulation at the Shangen acupoint for insomnia patients based on the fact that EEG signals are time series. Research subjects are 30 patients with primary insomnia from the Department of Sleep Medicine at the Rehabilitation Hospital affiliated with Fujian University of Traditional Chinese Medicine. Potential candidates for the study were screened and fully informed of the study. Eligible participants received SA intervention for 10 minutes, and EEG signals were collected for 10 minutes at each state, including prointervention (resting state), intervention (intervention state), and postintervention (postintervention state). The data were conducted using nonlinear dynamics (including Approximate Entropy (ApEn) and Correlation Dimension (CD)) and multivariate statistics.

This clinical trial was carried out in accordance with the Declaration of Helsinki and reviewed and approved by the Medical Ethics Committee Board of the rehabilitation hospital affiliated with Fujian University of Traditional Chinese Medicine, Fuzhou, Fujian, China (number: 2017KY-001-01). All of the participants need to sign the written informed consent form before trial. The whole experiment was operated and recorded by one experimenter.

\subsection{Participants and Recruitment}

2.2.1. Inclusion Criteria. Patients meeting all of the following criteria were enrolled in the study:

(1) Patient has a Pittsburgh Sleep Quality Index (PSQI) score $\geq 6$ and an insomnia history $\geq 1$ month, and symptoms of insomnia persisting $\geq 3$ days per week.

(2) Patient has an incubation period of falling asleep at night $\geq 30$ minutes and a total sleep time $<6$ hours.

(3) Patient meets The Chinese Classification and the Diagnose Criterion of Mental Disorder (CCMD-3) [27]. 


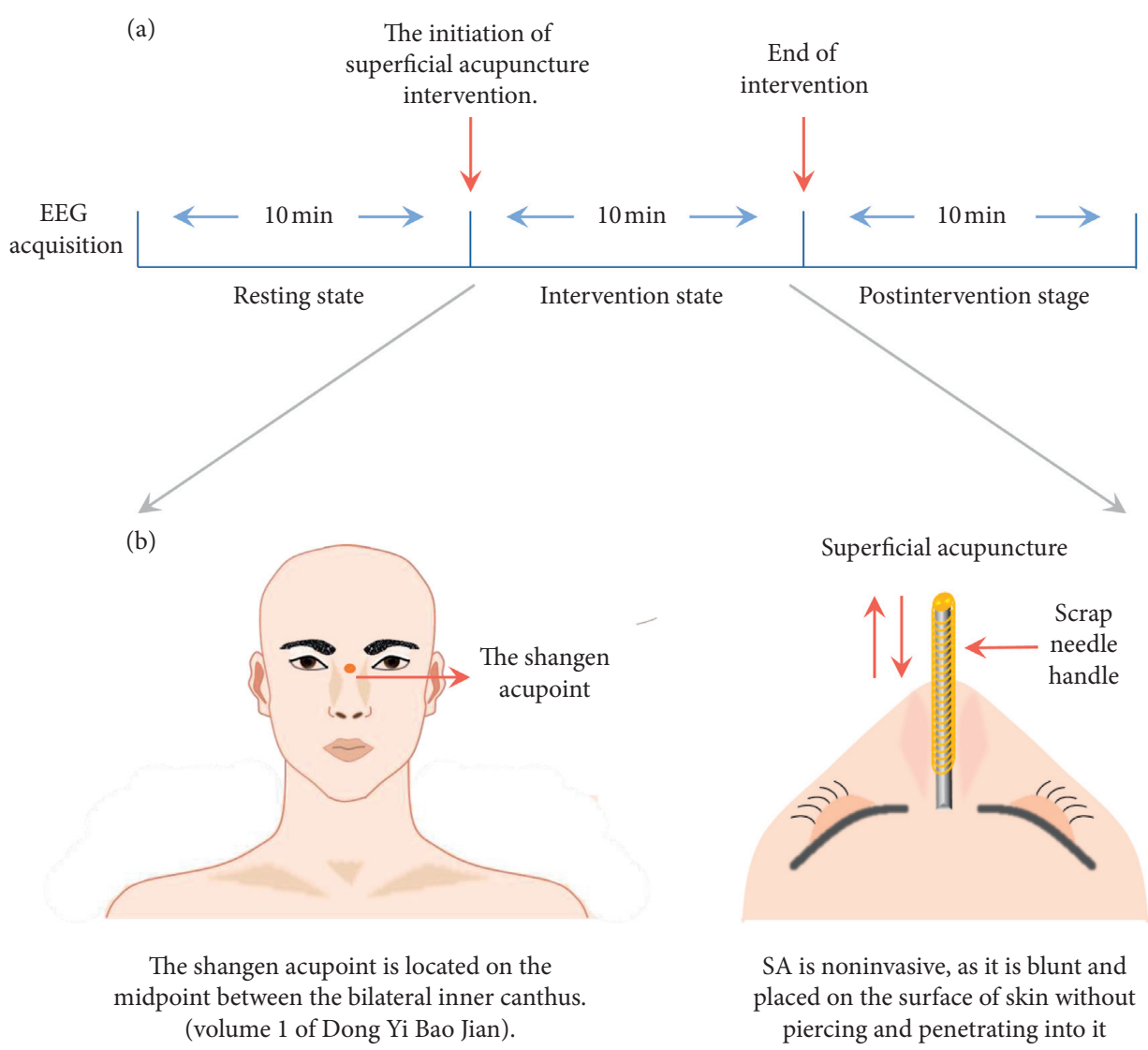

FIGURE 1: Flow diagram showing the intervention programme. (a) Experiment design of EEG measurement. (b) The location of the Shangen acupoint and introduction of SA.

(4) Patient meets the DSM-5 (Diagnostic and Statistical Manual of Mental Disorders, 5th Edition) diagnostic criteria for insomnia [28].

(5) Patient stops taking sedatives and tranquilizers, antidepressants and psychotropic drugs, and other drugs affecting sleep EEG signals.

(6) Patient has no history of head trauma, epilepsy, head metal implantation, or pacemaker implantation.

(7) Male and female patients between the ages of 18 and 75 years (inclusive) were eligible for participation.

(8) Patient provides signed informed consent.

2.2.2. Exclusion Criteria. Patients meeting any of the following criteria were excluded from the study:

(1) Patient suffers from physical or mental disorders that result in progressive insomnia or other types of sleep disorders.

(2) Patient works shifts involving changes in day/night work schedule that impacts circadian rhythm.

(3) Patient is pregnant, breast-feeding, or preparing to become pregnant.

(4) Patient has severe respiratory insufficiency and/or is given sedatives for 1 week.
(5) Patient has cardiovascular, endocrine, viscera, and/ or hematopoietic diseases.

(6) Patient is an alcoholic or psychotropic substance abuser.

(7) Patient is noncompliant with the treatment or study protocol.

\subsubsection{Withdrawal or Dropout Criteria}

(1) The participant has an adverse event caused by this study

(2) The participant requires to drop out.

We included 30 patients with insomnia in the study following the inclusion and exclusion criteria, all of whom were right-handed. There were 16 female cases and 14 male cases, between 28 and 73 years old. The flow diagram was performed in Figure 2.

2.3. Intervention. All of the SA treatments were completed by one acupuncturist. A piece of cotton was wrapped around the needle tip in order to reduce pain caused by the needle tip contacting the skin. Then it is gently put on the Shangen acupoint, which was selected based on the theory of acupuncture and traditional Chinese medicine (TCM), according to volume 1 of Dong Yi Bao Jian (Dongui Bogam). 


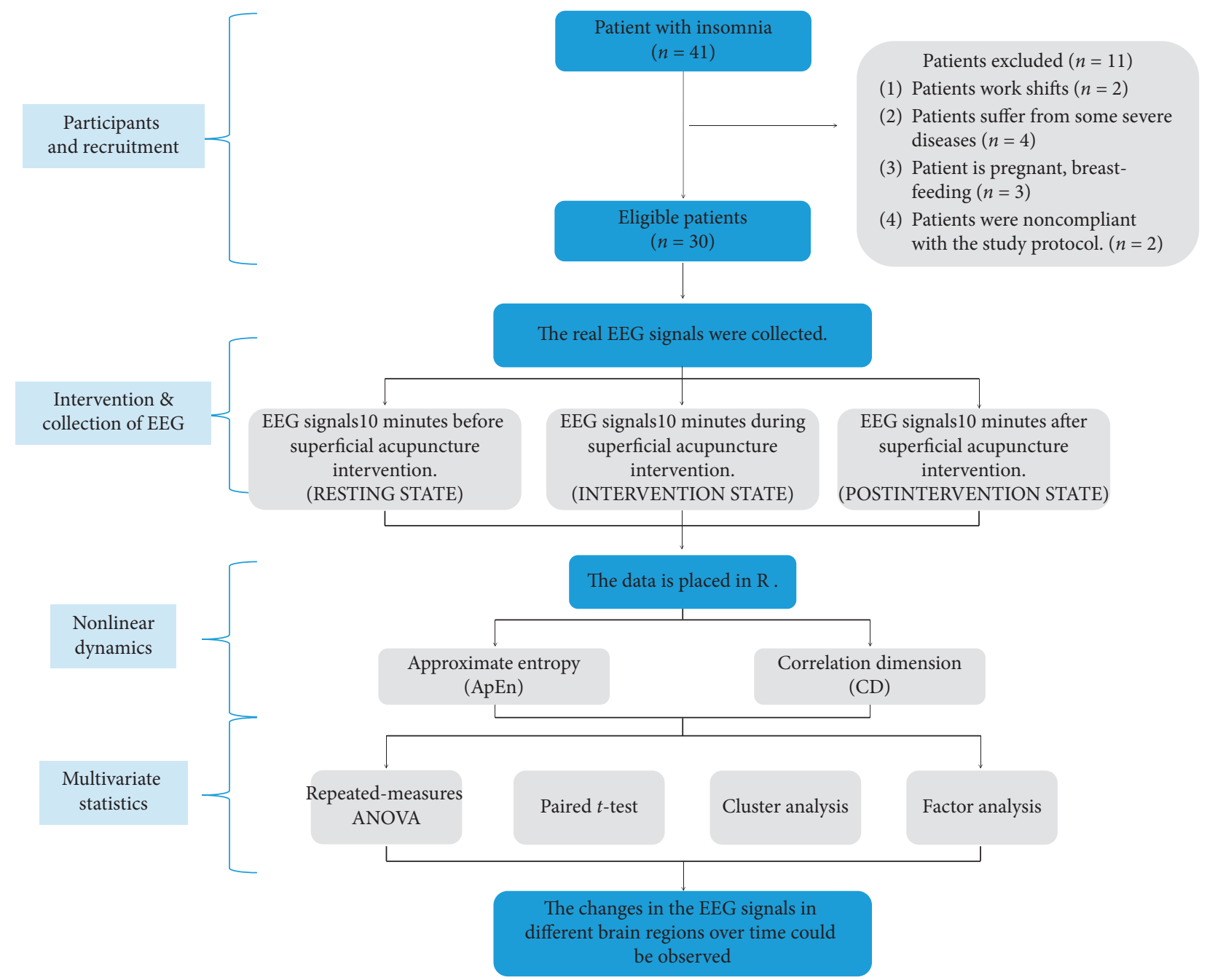

Figure 2: Flow chart.

This acupoint is located on the midpoint between the bilateral inner canthus. As previously described, the acupuncturist held the needle handle with two right-hand fingers (index finger and middle finger), and scraped the needle handle at a uniform velocity for 10 minutes with his or her thumb (see Figure 1(b)).

2.4. EEG Recording and Preprocessing. This trial was conducted in a quiet, shielded room. The participants could not hear or see any distractions. EEG electrodes were placed according to the 10-20 international standard lead system (see Figure 3). The EEG signals were recorded using a 16channel EEG from Beijing Upson Industry and Trade co., LTD. The data were acquired with the ZN16E high-frequency EEG signal amplifier, a pass-band filter of $0.3-100 \mathrm{~Hz}$, and a mode/digital conversion digit of 12 bits. The sampling rate was $128 \mathrm{~Hz}$ (sampling interval $0.07 \mathrm{~s}$ ). All electrodes were referenced to the earlobes, and 11000 data points were selected for each electrode.

When the instrument was in position, the subjects were asked to rest quietly with their eyes closed for $10 \mathrm{~min}$ before the data collection.

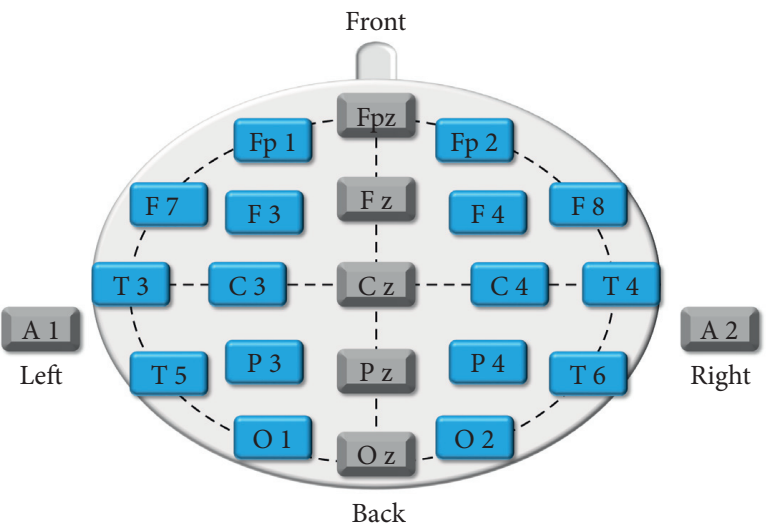

FIGURE 3: Schematic drawing of electrode positions.

(1) Each subject collected 10 min of EEG data before SA intervention (resting state)

(2) Each subject collected 10 min of EEG data during SA intervention at Shangen acupoint (intervention state) 
(3) Each subject collected 10 min of EEG data after SA intervention (postintervention state)

2.5. Data Processing. The data processing was performed using the $R$ programming language (version 3.6.3). As an open-source statistical language and a data analysis tool, $R$ was first developed in 1993 by Ross Ihaka and Robert Gentleman at the University of Auckland [29], which is an interpretive language for statistics and graphics with efficient data processing and storage abilities [30].

Approximate Entropy (ApEn), introduced by Pincus $[31,32]$, is a measure of data regularity, that is, the conditional probability of similarity vector which maintains its similarity when it increases from $m$ to $m+1$ dimension. Its physical meaning is the size of the probability of generating a new pattern in a time series as the dimension changes ApEn measures the volatile trend of the research object, which is determined by the complexity of time series. The more complex the time series is, the higher the probability of generating new patterns will be and the larger the corresponding ApEn; this also reflects a higher degree of brain activity in terms of EEG [23,33]. The formula is defined as follows:

$$
\operatorname{ApEn}(m, r)=\lim _{n \longrightarrow \infty}\left[\varphi^{m}(r)-\varphi^{m+1}(r)\right] .
$$

In this study, one ApEn ( $m=2, R=0.2 \mathrm{sd})$ is calculated from every 100 data using the "pracma" command in $R$. Then, the mean values of ApEn are taken from each electrode.

In addition, Grassberger and Procaccia introduced CD, a geometric measure of dynamic complexity that can be used to estimate the size of phase space [34]. In terms of an EEG, the $\mathrm{CD}$ represents the degrees of freedom of human brain activity; the decreasing $\mathrm{CD}$ values indicate that the components of the brain are continuously coupled or that the previously active parts begin to become inactivated [19]. The formula is defined as follows:

$$
\mathrm{CD}=\lim _{r \longrightarrow 0}\left[\frac{\ln C(r)}{\ln r}\right] .
$$

Data were processed using $R$ software, "nonlinear series" program.

2.6. Statistical Analysis. Data were analyzed using SPSS, version 22.0 (SPSS Inc). All statistical analyses were twotailed tests and the level of significance was 0.05. Data were presented as mean values \pm SD and $95 \%$ confidence intervals (CI) of the mean or frequencies. Measurements were analyzed by two-way repeated-measures ANOVA (time), paired $T$-test, cluster analysis, and factor analysis.

\section{Results}

3.1. Repeated-Measures ANOVA Results. Repeated-measures ANOVA for ApEn (Figure 4(a)), $F=0.786, p=0.776$ $>0.05$ and repeated-measures ANOVA for CD (Figure 4(b)), $F=0.379, p=0.999>0.05$.
3.2. Paired T-Test Results. The paired $t$-test results of ApEn and CD are shown in Table 1. The number with ${ }^{*}$ was the $p$ value less than 0.05 . That is, the ApEn values of electrodes $\mathrm{O} 2$ at the postintervention state decreased, compared with resting state $(p<0.05)$. The electrodes $\mathrm{O} 2$ were located in the right occipital lobe.

3.3. Cluster Analysis Results. The dendrograms on the left of Figures 5 and 6 show the cluster analysis results of ApEn and $\mathrm{CD}$. We extracted the cluster analysis results and drew them onto a schematic of electrode positions for visualization and better understanding, with blue representing normal control and red representing cluster analysis results.

The cluster analysis of ApEn revealed that the electrodes Fp2 at the resting state (Figure 5(a)), T6 at the intervention state (Figure 5(b)), and $\mathrm{O} 2$ at the postintervention state (Figure 5(c)) were the highest-ranking cluster. And the cluster analysis of $\mathrm{CD}$ indicated that the electrodes $\mathrm{O} 2$ at three states (Figure 6) was the only highest-ranking one.

3.4. Factor Analysis. Using the SPSS factor analysis procedure, we carried out a Principal Component Factor Analysis and selected the common factors which were cumulative contribution rate of the common factors $\geq 75 \%$ and the eigenvalues $\geq 1$. The result diagrams on the left of Figures 7 and 8 show the factor analysis results of ApEn and CD.

Just as with the cluster analysis described above, we extracted the factor analysis results and drew them onto a schematic of electrode positions for visualization and better understanding, with blue representing normal control and red representing factor analysis results.

The factor analysis of ApEn revealed that the electrodes Fp2, T3, C4, O2 at the resting state (Figure 7(a)), F3, C4, T4 at the intervention state (Figure 7(b)), and F7, F8, C3, P3 at the postintervention state (Figure $7(\mathrm{c})$ ) were more prominent.

And the factor analysis of $\mathrm{CD}$ indicated that the electrodes Fp1, Fp2, F3, F4, F7, F8, T3, T4, T5, T6, C3, C4, P3, and $\mathrm{P} 4$ at the resting state (Figure 8(a)), FP1, FP2, F3, F4, F7, F8, T3, T4, T5, T6, C3, C4, and P3 at the intervention state (Figure 8(b)), and Fp1, Fp2, F3, F4, F7, and F8 at the postintervention state (Figure $8(\mathrm{c})$ ) were more prominent.

\section{Discussion}

Fundamental principles of metabolic coupling between different brain regions have recently been studied using neuroimaging techniques (fMRI, PET), proving that there is a specific relationship between acupoints and brain regions $[35,36]$. This relationship is not a one-to-one correspondence between an acupoint and a single functional brain region, but multidimensional interactions between an acupoint and multiple distributed areas of the brain.

As the high spatial but low temporal correlation [37], the neuroimaging techniques could not detect significant changes in the connectivity of these neural networks structures under certain circumstance [19]. Some researches demonstrated that the temporal dynamics of an ongoing 


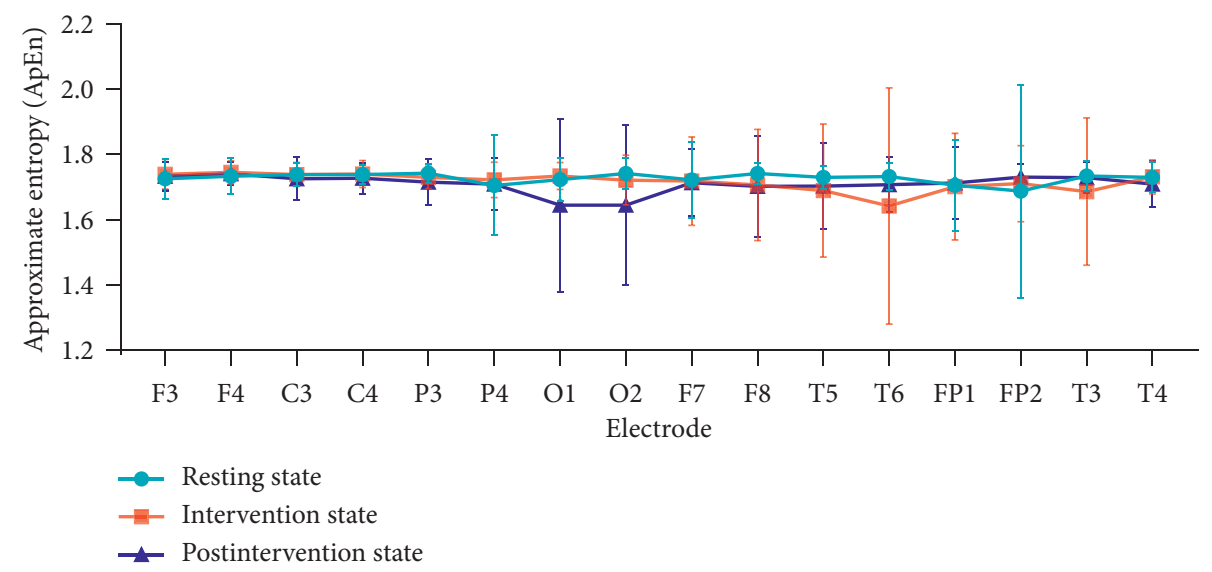

(a)

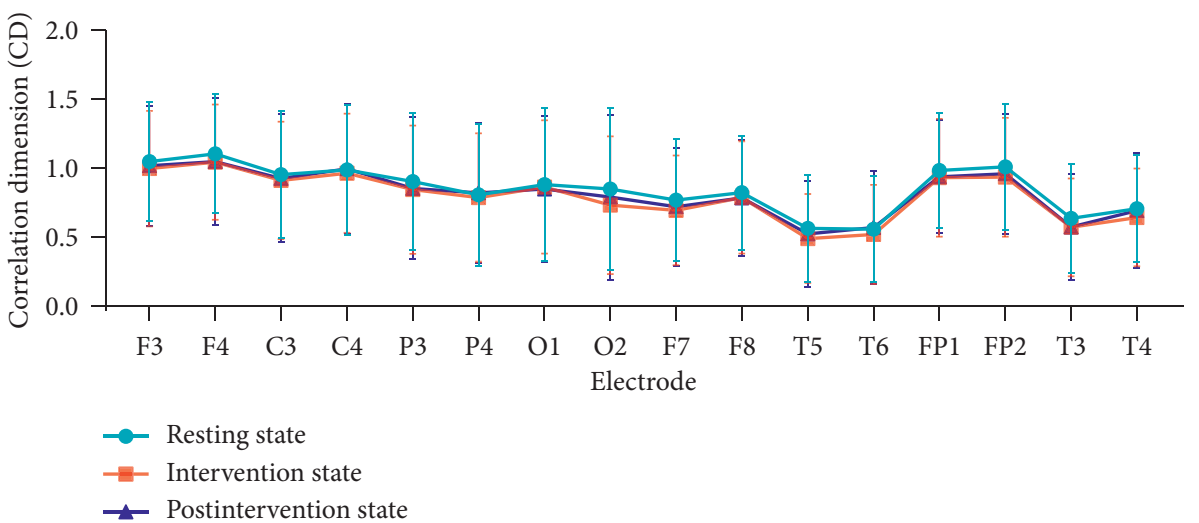

(b)

FIGURE 4: Repeated-measures ANOVA results.

TABle 1: Paired $t$-test results (mean $\pm \mathrm{SD}, n=30$ ).

\begin{tabular}{|c|c|c|c|c|c|c|}
\hline \multicolumn{7}{|l|}{ Mean \pm SD } \\
\hline \multirow{2}{*}{ Electrode } & \multicolumn{3}{|c|}{ Approximate entropy (ApEn) } & \multicolumn{3}{|c|}{ Correlation dimension (CD) } \\
\hline & Resting state & Intervention state & Postintervention state & Resting state & Intervention state & Postintervention state \\
\hline F3 & $1.73 \pm 0.06$ & $1.75 \pm 0.05$ & $1.74 \pm 0.04$ & $1.06 \pm 0.43$ & $1.01 \pm 0.42$ & $1.03 \pm 0.44$ \\
\hline $\mathrm{F} 4$ & $1.74 \pm 0.05$ & $1.75 \pm 0.04$ & $1.75 \pm 0.04$ & $1.12 \pm 0.43$ & $1.06 \pm 0.42$ & $1.06 \pm 0.46$ \\
\hline C3 & $1.75 \pm 0.04$ & $1.75 \pm 0.04$ & $1.73 \pm 0.07$ & $0.97 \pm 0.46$ & $0.93 \pm 0.43$ & $0.94 \pm 0.46$ \\
\hline $\mathrm{C} 4$ & $1.75 \pm 0.03$ & $1.75 \pm 0.04$ & $1.73 \pm 0.05$ & $1.00 \pm 0.47$ & $0.98 \pm 0.43$ & $1.01 \pm 0.47$ \\
\hline P3 & $1.75 \pm 0.03$ & $1.74 \pm 0.03$ & $1.72 \pm 0.07$ & $0.92 \pm 0.50$ & $0.86 \pm 0.47$ & $0.87 \pm 0.52$ \\
\hline $\mathrm{P} 4$ & $1.71 \pm 0.15$ & $1.73 \pm 0.05$ & $1.72 \pm 0.08$ & $0.82 \pm 0.52$ & $0.80 \pm 0.46$ & $0.84 \pm 0.51$ \\
\hline $\mathrm{O} 1$ & $1.73 \pm 0.07$ & $1.74 \pm 0.04$ & $1.65 \pm 0.26$ & $0.90 \pm 0.56$ & $0.88 \pm 0.48$ & $0.86 \pm 0.53$ \\
\hline $\mathrm{O} 2$ & $1.75 \pm 0.05$ & $1.73 \pm 0.08$ & $1.65 \pm 0.24^{*}$ & $0.87 \pm 0.59$ & $0.75 \pm 0.50$ & $0.81 \pm 0.60$ \\
\hline F7 & $1.73 \pm 0.12$ & $1.73 \pm 0.14$ & $1.72 \pm 0.10$ & $0.78 \pm 0.44$ & $0.71 \pm 0.40$ & $0.74 \pm 0.43$ \\
\hline F8 & $1.75 \pm 0.03$ & $1.71 \pm 0.17$ & $1.71 \pm 0.16$ & $0.84 \pm 0.41$ & $0.80 \pm 0.41$ & $0.80 \pm 0.42$ \\
\hline T5 & $1.74 \pm 0.04$ & $1.70 \pm 0.20$ & $1.71 \pm 0.13$ & $0.58 \pm 0.39$ & $0.51 \pm 0.32$ & $0.54 \pm 0.38$ \\
\hline T6 & $1.74 \pm 0.04$ & $1.65 \pm 0.36$ & $1.72 \pm 0.08$ & $0.57 \pm 0.39$ & $0.54 \pm 0.36$ & $0.89 \pm 0.41$ \\
\hline FP1 & $1.71 \pm 0.14$ & $1.71 \pm 0.16$ & $1.72 \pm 0.11$ & $1.00 \pm 0.41$ & $0.95 \pm 0.43$ & $0.95 \pm 0.41$ \\
\hline FP2 & $1.70 \pm 0.33$ & $1.72 \pm 0.12$ & $1.74 \pm 0.04$ & $1.02 \pm 0.46$ & $0.95 \pm 0.43$ & $0.97 \pm 0.44$ \\
\hline T3 & $1.74 \pm 0.05$ & $1.69 \pm 0.23$ & $1.74 \pm 0.05$ & $0.65 \pm 0.40$ & $0.59 \pm 0.36$ & $0.59 \pm 0.38$ \\
\hline $\mathrm{T} 4$ & $1.74 \pm 0.05$ & $1.74 \pm 0.05$ & $1.72 \pm 0.07$ & $0.72 \pm 0.39$ & $0.66 \pm 0.35$ & $0.71 \pm 0.42$ \\
\hline
\end{tabular}

"stream of consciousness" is much faster than the time resolution of blood oxygen lever-dependent (BOLD) [38]. Therefore, in spite of its low spatial resolution, the EEG is more appropriate for obtaining the changes in different brain regions over time to understand the central mechanisms of SA intervention. 


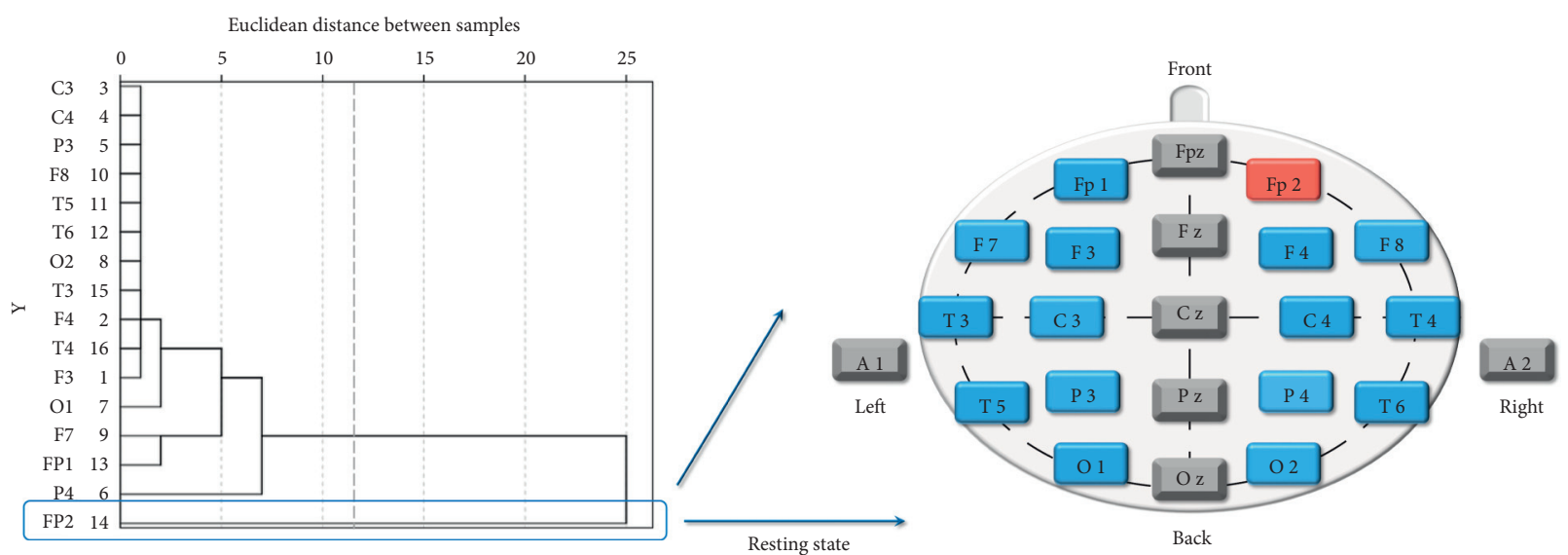

(a)
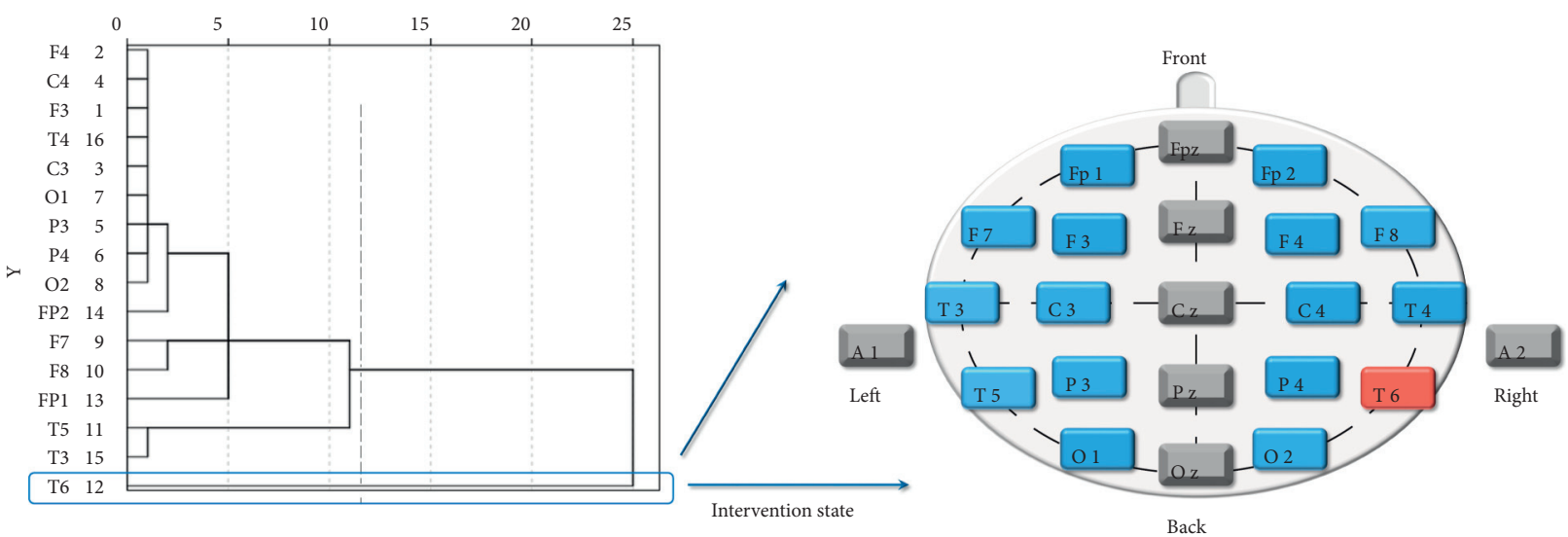

(b)
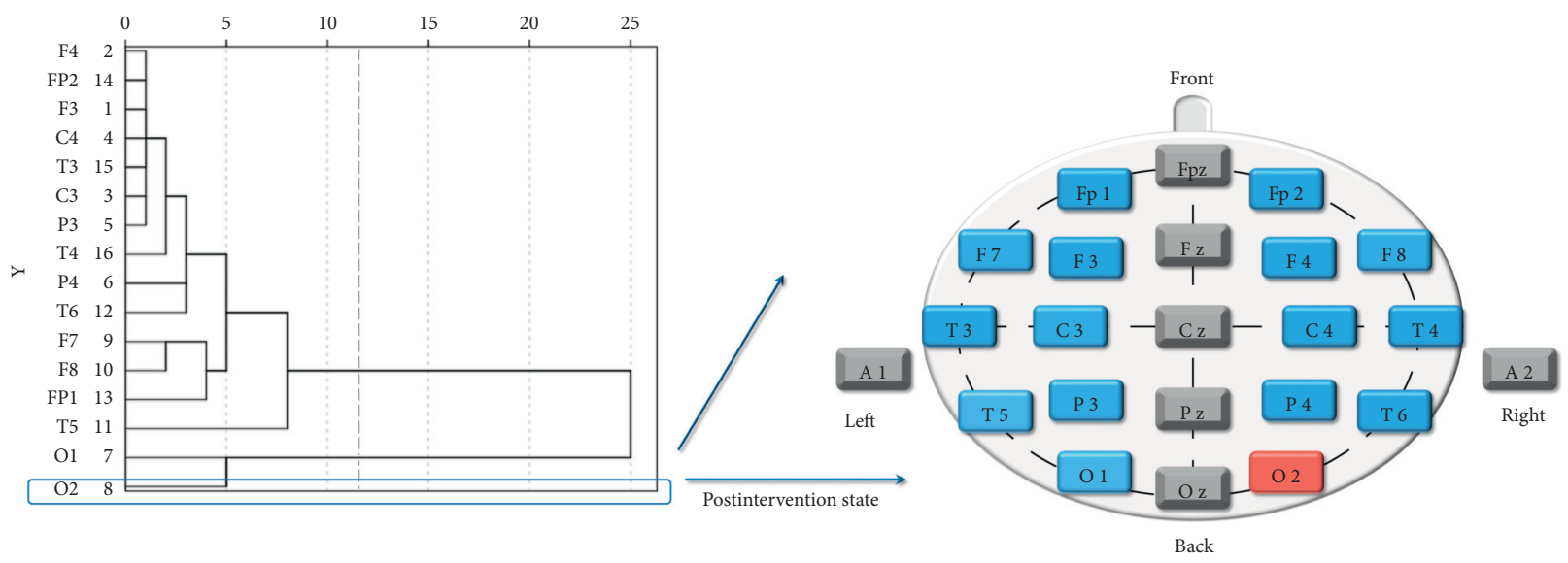

(c)

Figure 5: Cluster analysis diagram of ApEn at the three states. (a) Resting state, (b) intervention state (c) postintervention state.

However, as the majority of physiological signals are by nature, nonstationary, and nonlinear, it is difficult to directly observe the effects of SA on the body over time using EEG [39]. There is no doubt that mathematics is integral to the study of biological systems that can be used to quantitatively explore and explain some phenomena better than compared to only experimental observations [29]. Therefore, this study put forward a method of combining the nonlinear dynamics with multivariate statistics to analyze the data. Nonlinear dynamics can serve as a fruitful tool that quantifies the complexity of a time series. Therefore, it can be used to evaluate the nonlinear, unstable EEG [32]. Overall, the multivariate statistics can provide a productive approach for detecting the overall changes and connectivity in brain regions after SA intervention.

The results of repeated-measures ANOVA in Figure 4 indicated no statistical difference both in ApEn and CD, suggesting that the use of nonlinear analysis gave the 


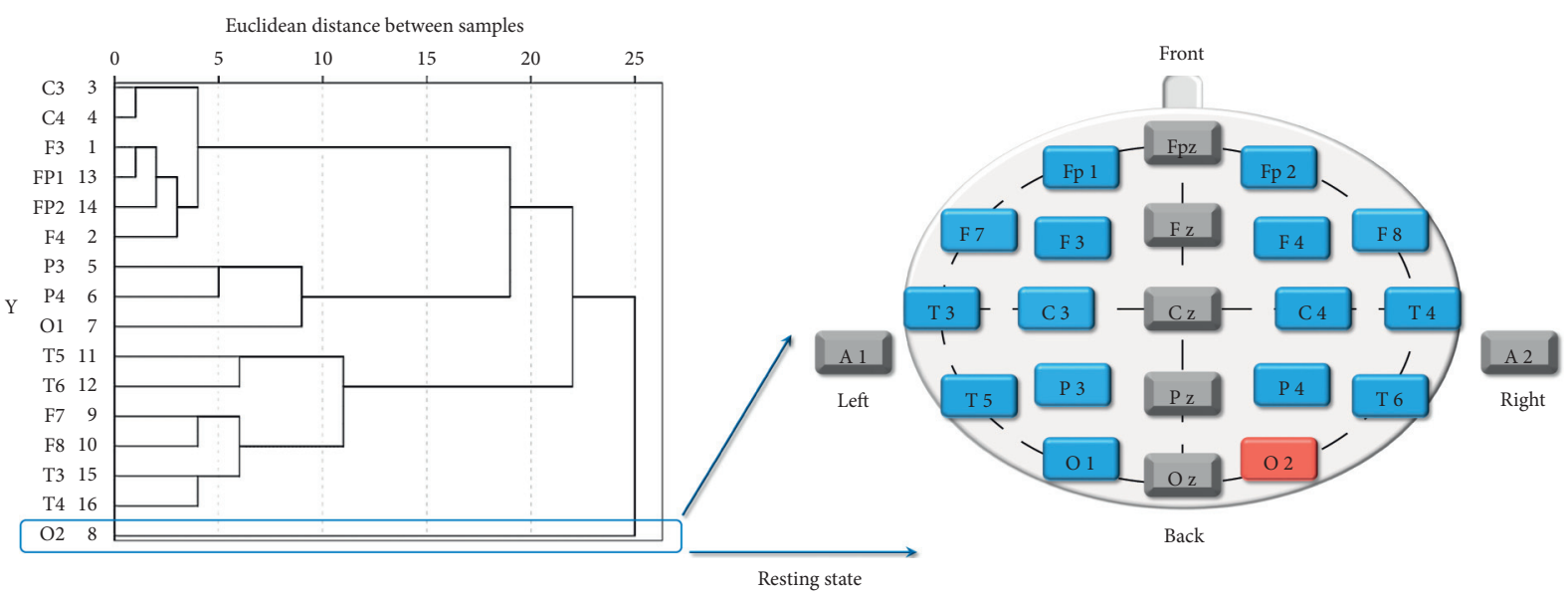

(a)

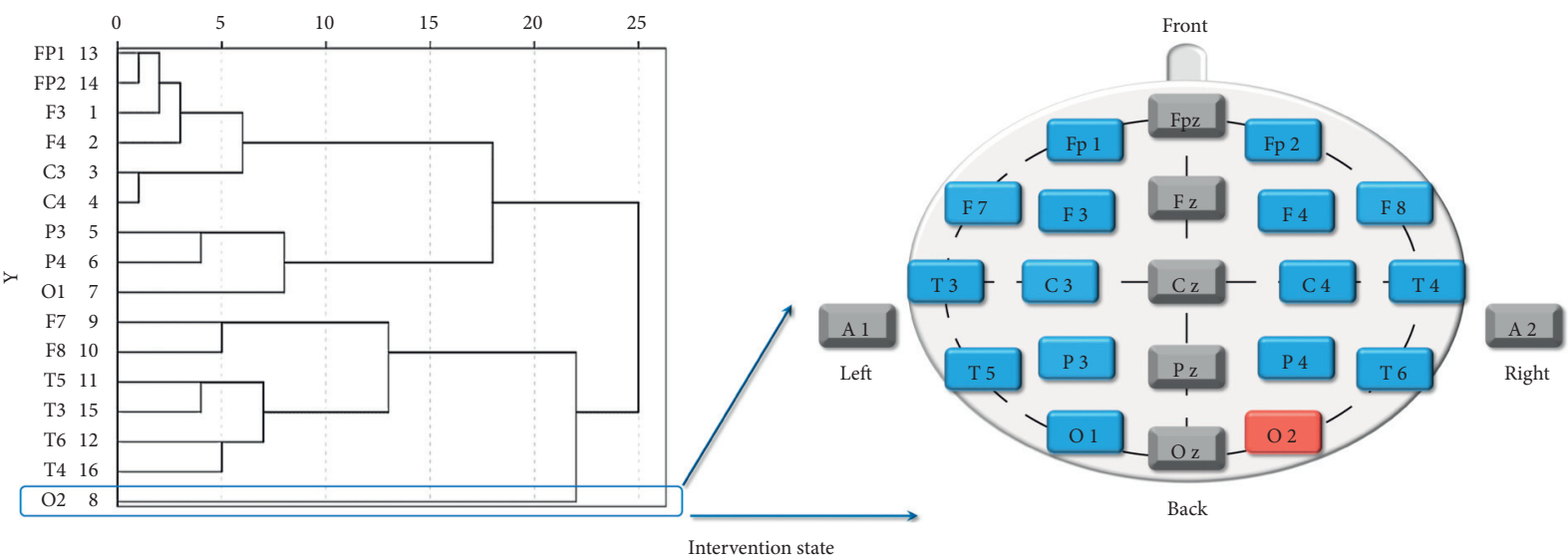

(b)

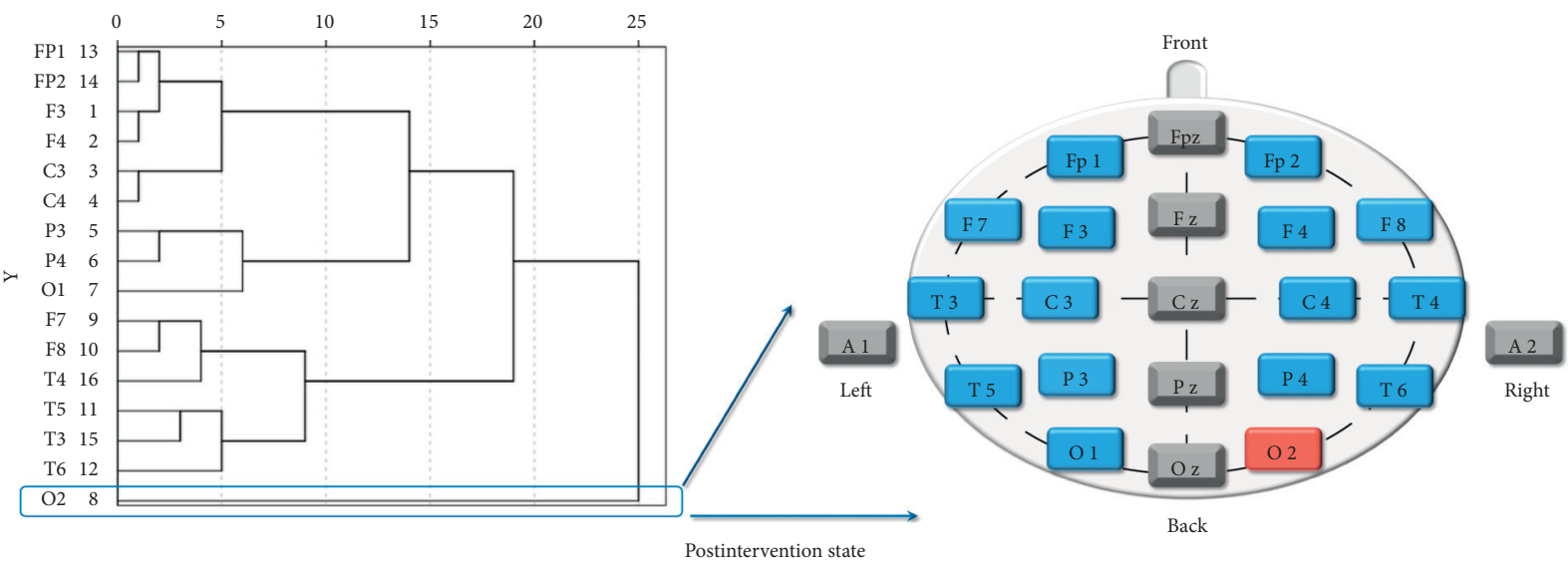

(c)

Figure 6: Cluster analysis diagram of $\mathrm{CD}$ at the three states. (a) Resting state, (b) intervention state, (c) postintervention state.

advantages to deal with the spectrum and complexity characteristics of the EEG signal and make it more consistent, synchronized, and stable. The cluster analysis results of ApEn were performed that the right prefrontal lobe (electrode Fp2) at the resting state was the highest-ranking one. That was consistent with other research. Some researchers found that compared to the control group, the beta1 power spectrum was lower in the prefrontal cortex in the insomnia group [40, 41]. Furthermore, we found that the SA stimulation affected the right posterior temporal lobe and then spread to the occipital lobe, and this result was in good agreement with the paired $t$-test results of ApEn, which showed the ApEn values of electrode $\mathrm{O} 2$ at postintervention state have decreased, compared with resting state $(p<0.05)$. The cluster analysis results of CD also showed the electrode $\mathrm{O} 2$ was the only highest-ranking one at three states. Some 

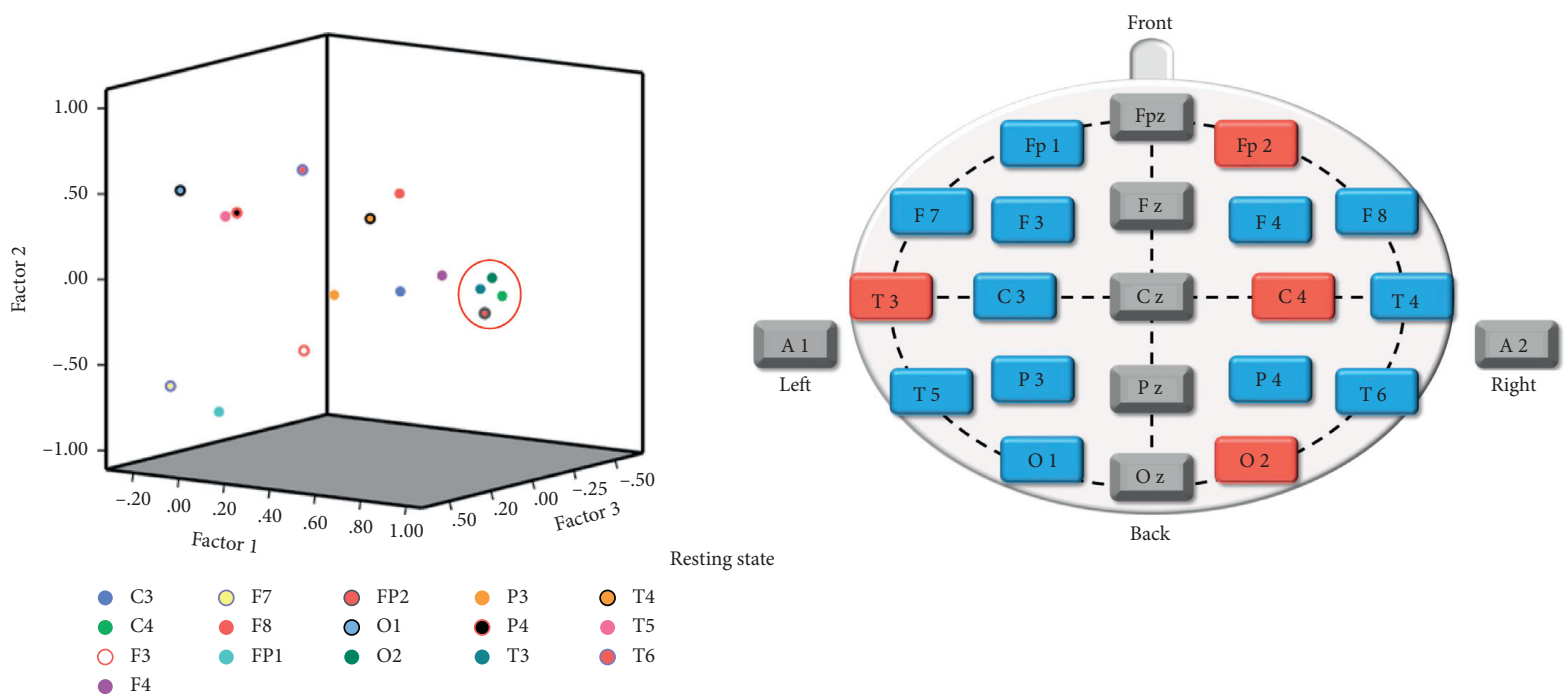

(a)
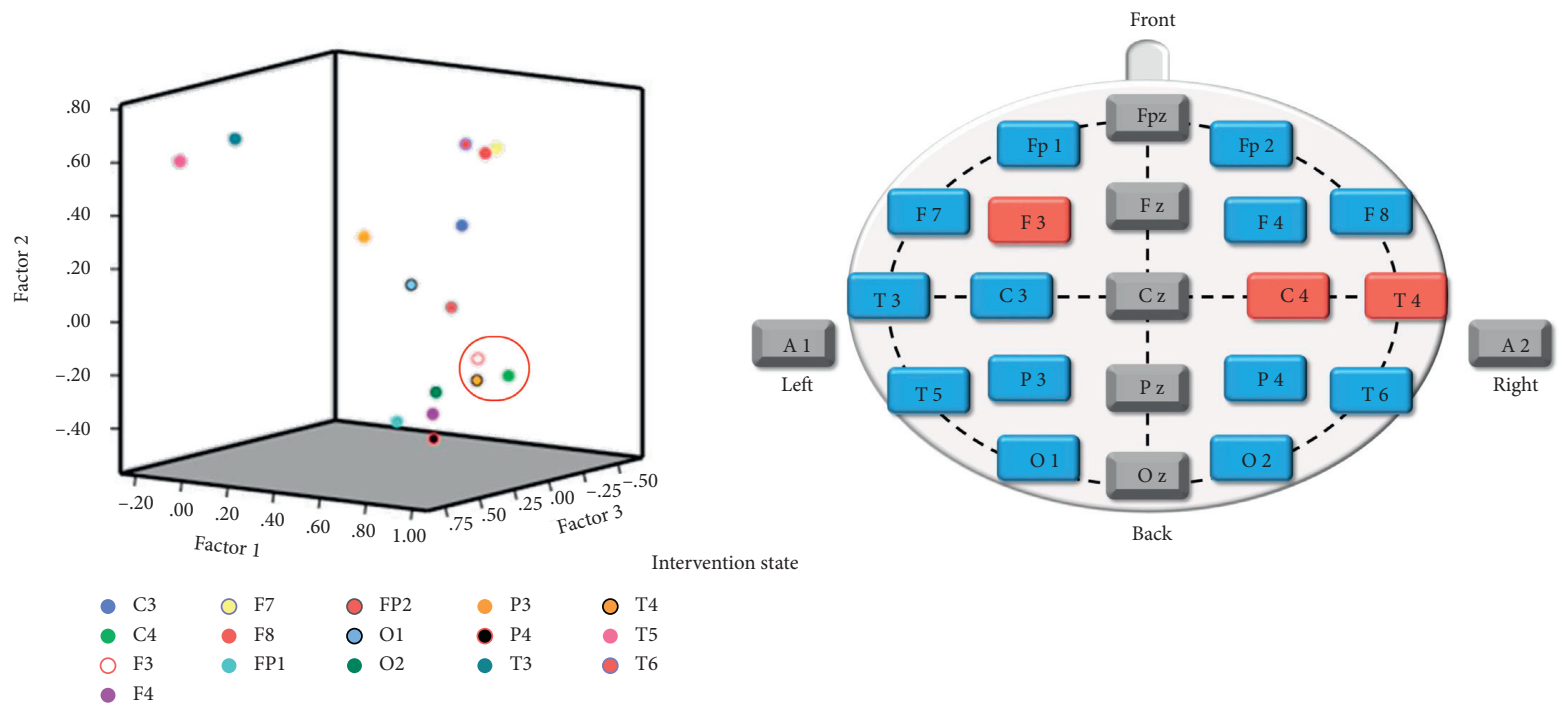

Intervention state

(b)
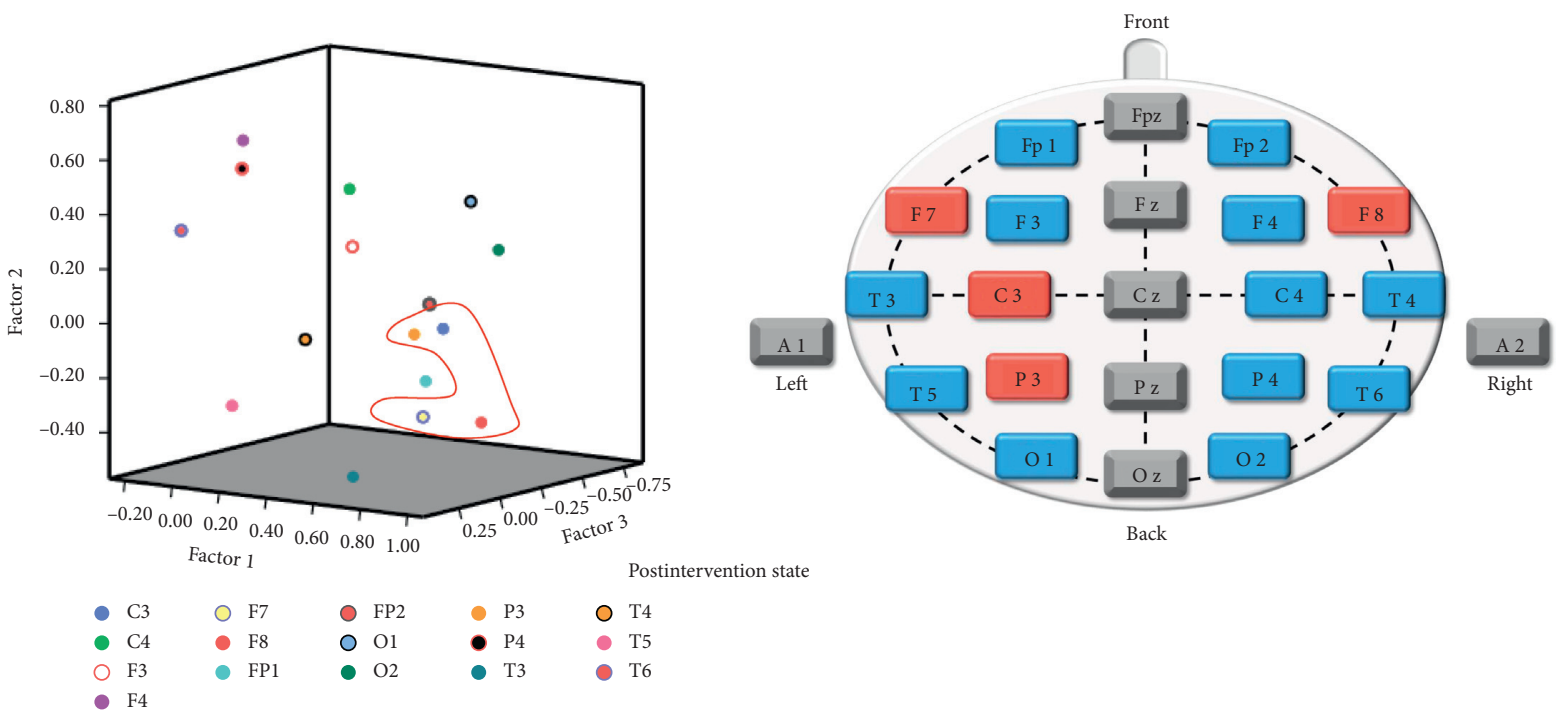

(c)

FIgURE 7: Factor analysis diagram of ApEn at the three states. (a) Resting state, (b) intervention state, (c) postintervention state. 

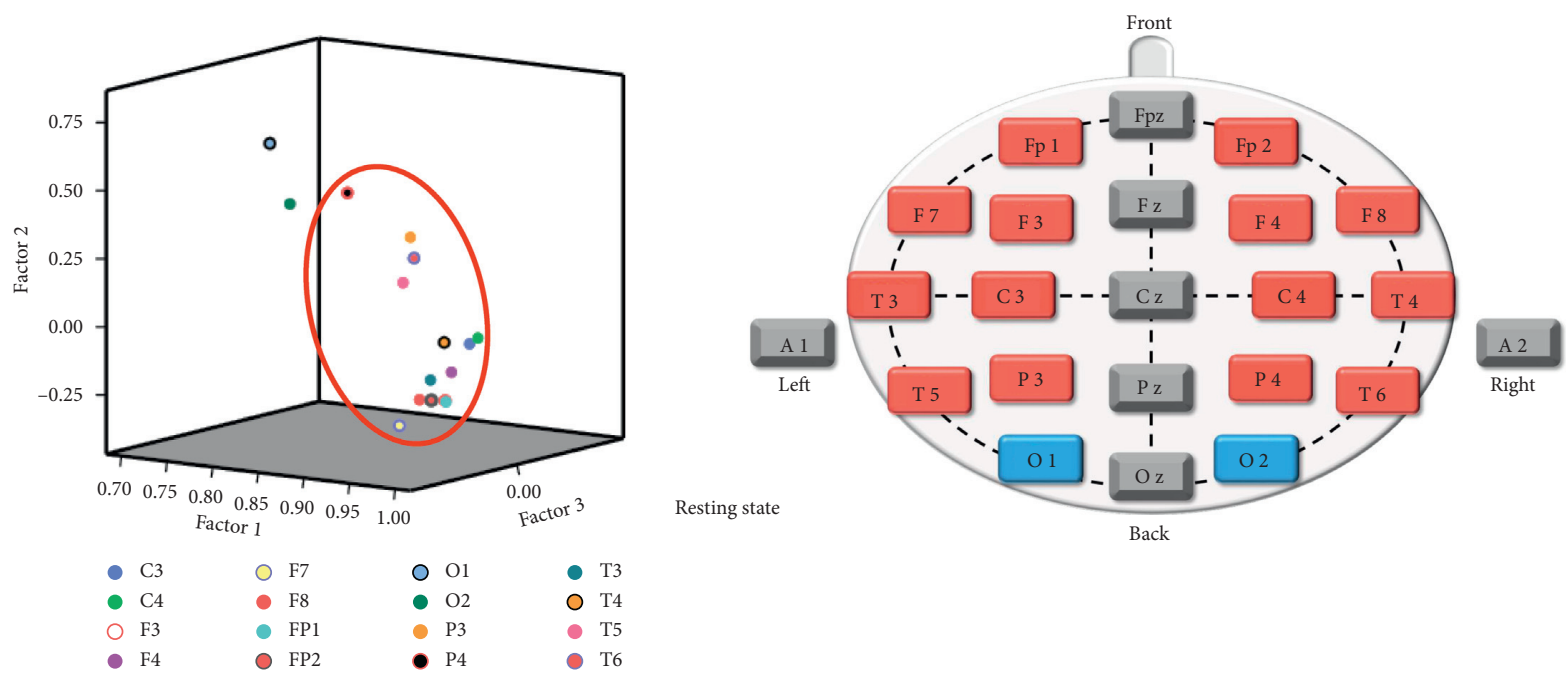

(a)
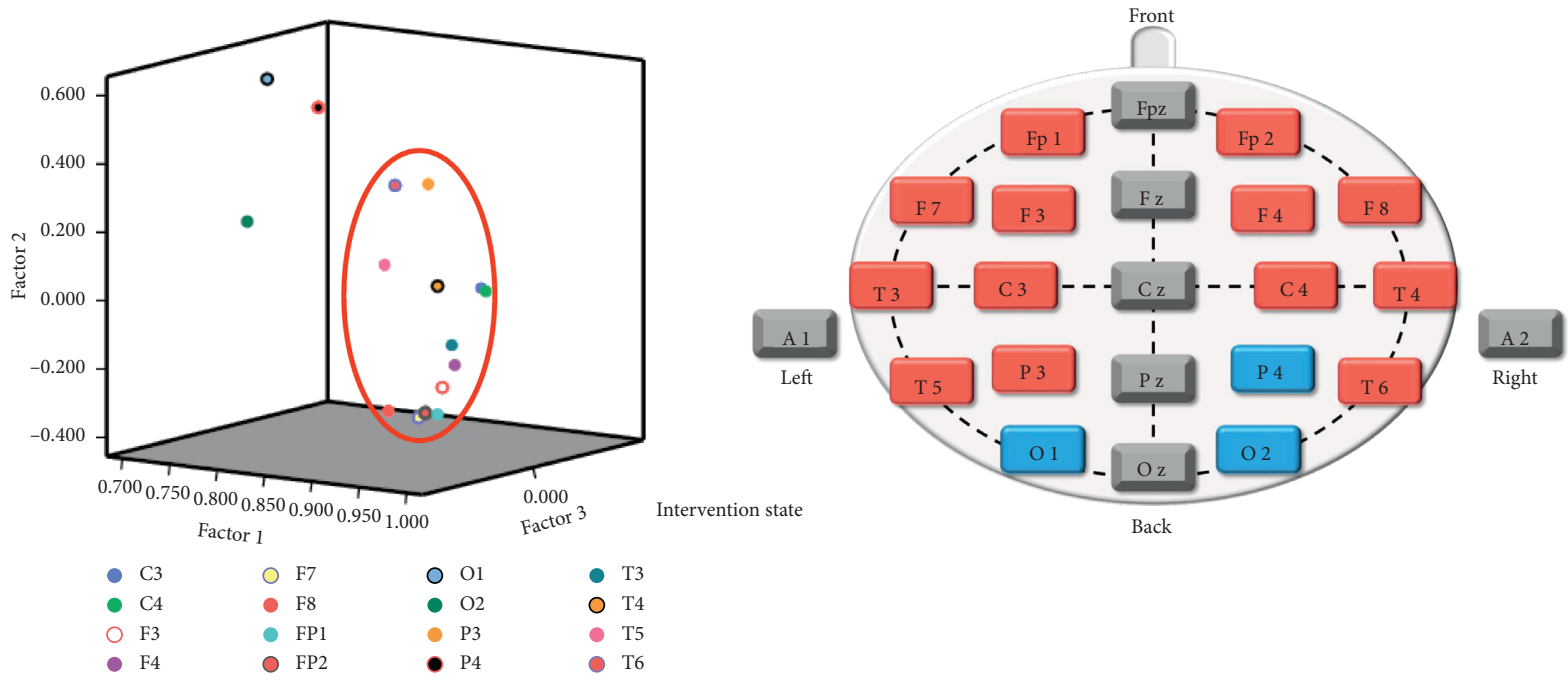

(b)
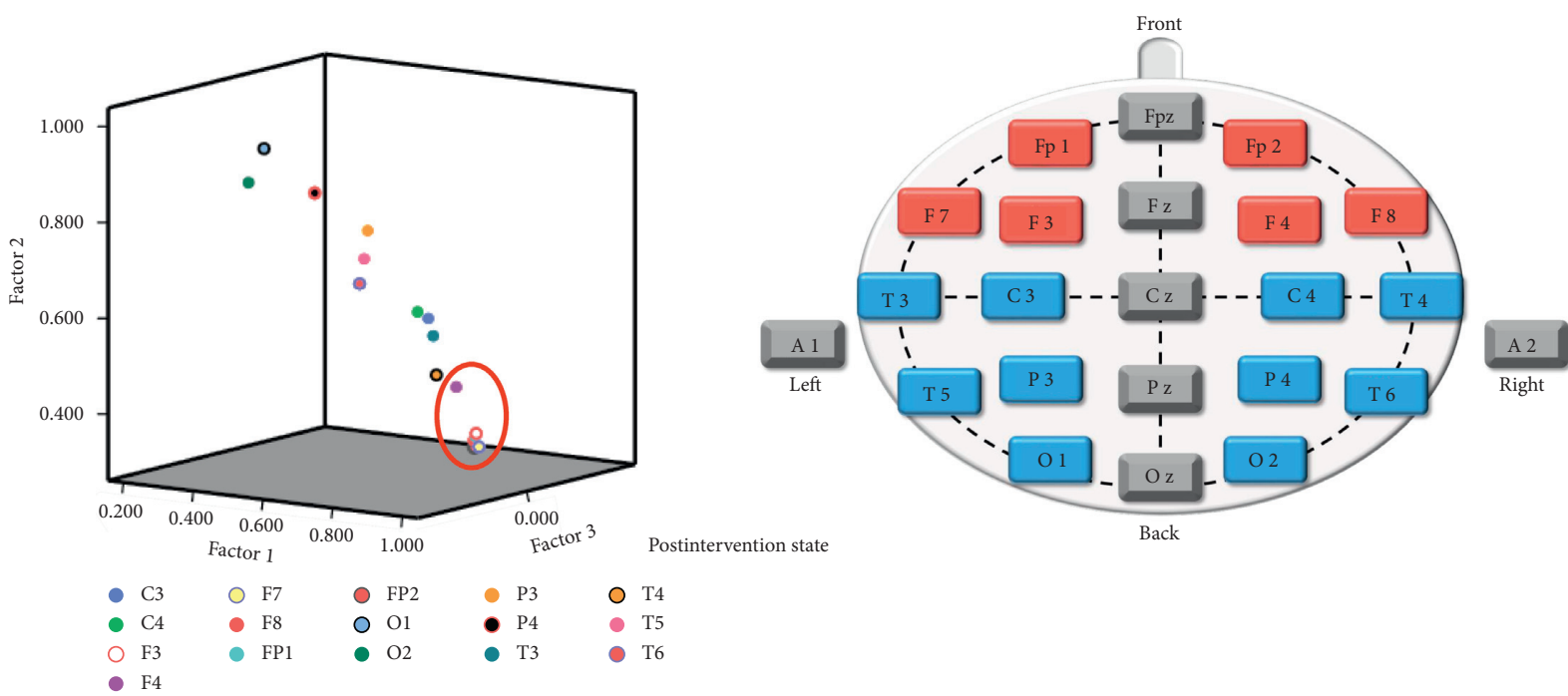

(c)

FIgURE 8: Factor analysis diagram of CD at the three states. (a) Resting state, (b) intervention state, (c) postintervention state. 


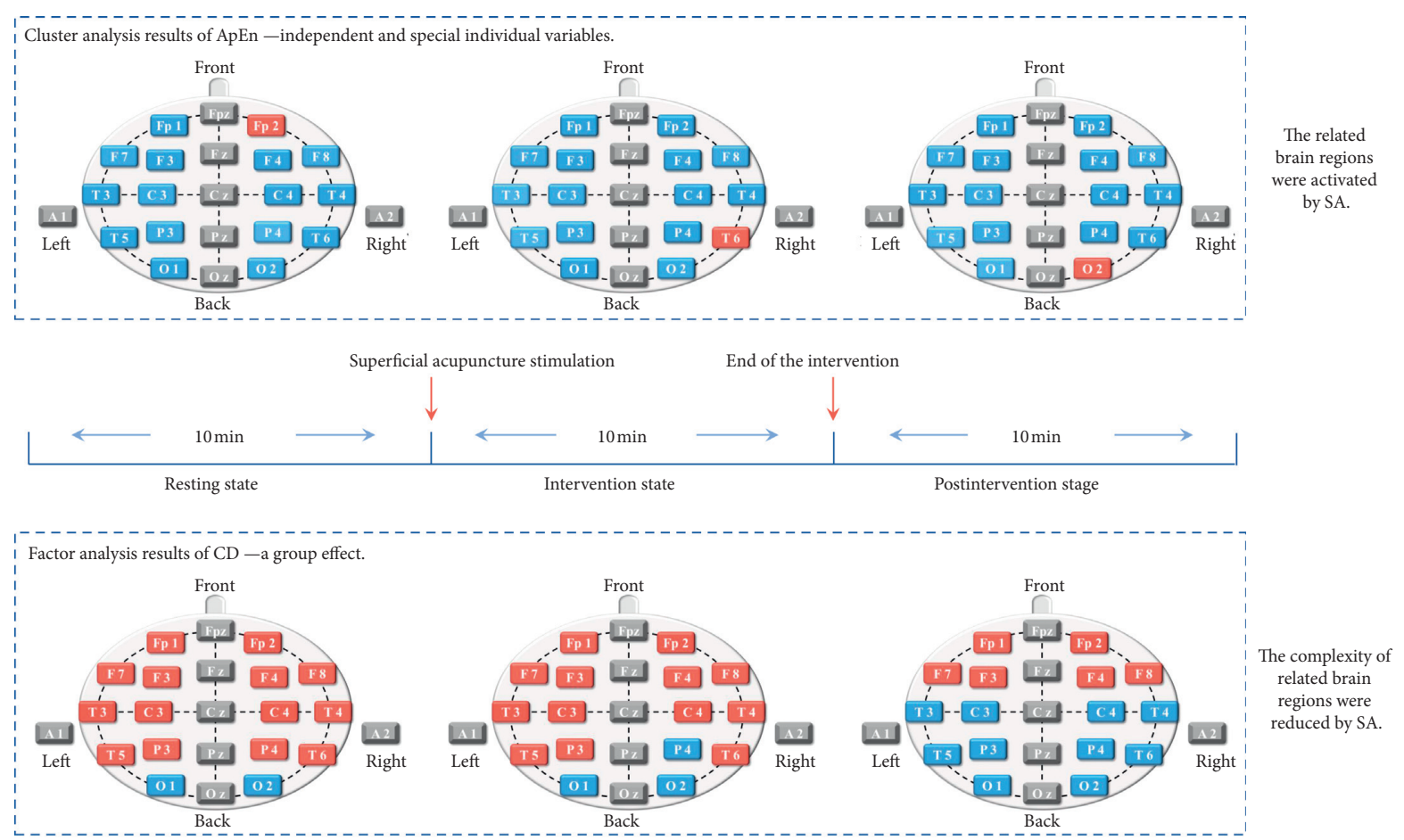

FIgURE 9: Schematic representation of the main findings from the study.

studies indicated that the mean occipital GABA/Cr ratio was significantly higher in the insomnia group than in the control group [42]. Therefore, we hypothesized that the cluster analysis of CD might highlight the abnormal EEG in the occipital lobe for insomniacs. In addition, the results of the factor analysis of CD performed that whole brain regions except the occipital lobes changed to the frontal poles, anterior temporal, and frontal lobes from pre-to postintervention.

Cluster analysis classifies individuals into classes and identifies special variables within a cluster that are more similar to each other than the variables contained in different clusters [43]. It aims to delimit specially activated EEG signals. In other words, its purpose is to observe changes in EEG caused by SA stimulation. In addition, by retaining the original variable information and decomposing the original information to classify potential categories, factor analysis can determine which variables form coherent subsets (factors) that are somewhat independent and that can reflect the overall information [44, 45]. Therefore, unlike cluster analysis, using factor analysis approaches aims to assess the internal structure. That is to say, the results of cluster analysis were independent and special individual variables. The results of factor analysis were a group effect, meaning a global alteration of brain state (see Figure 9).

Moreover, ApEn and CD are each considered to reflect different aspects of data. ApEn is an algorithm used to measure the complexity of time series [20]. We found that the cluster analysis results of ApEn might be associated with brain activation by SA stimulation, reflecting needlingspecific brain responses. And CD is one of the most basic quantitative indexes of chaotic time series used to measure the system of complexity by assessing attractor dimension in the reconstructed phase space [21], reflecting the correlation degree of EEG signals sequence itself [46]. Studies have shown that the magnitude of the $\mathrm{CD}$ reflected the frequency of activity of neurons in one particular brain region. The larger CD values, the more complex the neuron activity [47]. Therefore, based on the factor analysis results of CD, we hypothesized that one of the possible central mechanisms of SA intervention was the suppression of activity in the relevant brain regions, including the temporal, central, posterior temporal, and parietal lobes. It reduced the complexity of the brain regions involved. The obtained results were in good agreement with the medical report and MRI data, which demonstrated different insomnia-related heterotopic connectivity patterns in the right and left middle occipital/ posterior middle temporal gyrus [48]. Besides, we have not found the regularity in factor analysis results of ApEn, nor in the cluster analysis of CD. It may be relevant that the ApEn is more suitable for cluster analysis, whereas $\mathrm{CD}$ is more suitable to factor analysis, according to their characteristics.

In summary, based on the fact that EEG signals are time series, it is feasible to use nonlinear dynamics analysis and multivariate statistics to examine the effects of SA on the human brain. In this study, the cluster analysis of ApEn may be the characterization of specially activated EEG signals by SA. That is, patients' EEG changed from the right prefrontal lobe to the right posterior temporal lobe and finally to the right occipital lobe, before, during, and after the SA intervention. While the factor analysis of $C D$ may be the characterization of an overall alteration of brain state, showing that suppression of activity in the relevant brain regions, including the temporal, central, posterior temporal, and 
parietal lobes. We referred these changes to "the coupling effect among multiple brain regions." It means taking the temporal and spatial factors as parameters, the characteristics and connectivity of the overall changes between brain regions after intervention are revealed. We hope to apply this method to more studies on the mechanism of SA and acupuncture in the future, and it provides a new way to study the temporal and spatial characteristics of the influence of acupuncture on the central nervous system.

\section{Data Availability}

The datasets used and/or analyzed during the current study are available from the corresponding author upon reasonable request.

\section{Conflicts of Interest}

The authors declare that there are no conflicts of interest associated with this manuscript.

\section{Authors' Contributions}

SYQ performed data analysis and wrote the paper. DL designed the study and performed manuscript revision. $\mathrm{XZH}$ and YYY performed parts of the experiment. LLL, SL, $\mathrm{CHC}$, and ZXW performed manuscript revision. All authors approved the final version of this paper.

\section{Acknowledgments}

This work was financially supported by the "National Natural Science Foundation of China (NSFC)" (Grants nos. 82074521).

\section{References}

[1] W. Zhang, Z. Huang, and Y. Jin, “Acupuncture as a primary and independent treatment for a patient with chronic insomnia," Medicine, vol. 96, no. 52, p. e9471, 2017.

[2] K. F. Chung, W. F. Yeung, F. Y. Y. Ho, and T. K. Ng, "Dysfunctional cognition regarding sleep as a mediator of outcome following acupuncture for insomnia," Acupuncture in Medicine, vol. 36, no. 3, pp. 193-194, 2018.

[3] K. K. Gulia and V. M. Kumar, "Sleep disorders in the elderly: a growing challenge," Psychogeriatrics, vol. 18, no. 3, pp. 155-165, 2018.

[4] C. Baglioni, W. Regen, A. Teghen et al., "Sleep changes in the disorder of insomnia: a meta-analysis of polysomnographic studies," Sleep Medicine Reviews, vol. 18, no. 3, pp. 195-213, 2014.

[5] L. K. P. Suen, A. Molassiotis, S. K. W. Yueng, and C. H. Yeh, "Comparison of magnetic auriculotherapy, laser auriculotherapy and their combination for treatment of insomnia in the elderly: a double-blinded randomised trial," EvidenceBased Complementary and Alternative Medicine, vol. 2019, Article ID 3651268, 2019.

[6] K.-H. Han, S.-Y. Kim, and S.-Y. Chung, "Effect of acupuncture on patients with insomnia: study protocol for a randomized controlled trial," Trials, vol. 15, no. 1, p. 403, 2014.

[7] K.-F. Chung, W.-F. Yeung, B. Y.-M. Yu et al., "Acupuncture with or without combined auricular acupuncture for insomnia: a randomised, waitlist-controlled trial," Acupuncture in Medicine, vol. 36, no. 1, pp. 2-13, 2018.

[8] J. Guo, W. Huang, C.-Y. Tang, G.-L. Wang, F. Zhang, and L.-P. Wang, "Effect of acupuncture on sleep quality and hyperarousal state in patients with primary insomnia: study protocol for a randomised controlled trial," BMJ Open, vol. 6, no. 3, Article ID e009594, 2016.

[9] L. A. Gellis, A. Park, M. T. Stotsky, and D. J. Taylor, “Associations between sleep hygiene and insomnia severity in college students: cross-sectional and prospective analyses," Behavior Therapy, vol. 45, no. 6, pp. 806-816, 2014.

[10] D. Riemann, K. Spiegelhalder, B. Feige et al., "The hyperarousal model of insomnia: a review of the concept and its evidence," Sleep Medicine Reviews, vol. 14, no. 1, pp. 19-31, 2010.

[11] W. Zheng, Z. Su, X. Liu et al., "Modulation of functional activity and connectivity by acupuncture in patients with Alzheimer disease as measured by resting-state fMRI," PLoS One, vol. 13, no. 5, Article ID e0196933, 2018.

[12] J. Jittiwat, "Baihui point laser acupuncture ameliorates cognitive impairment, motor deficit, and neuronal loss partly via antioxidant and anti-inflammatory effects in an animal model of focal ischemic stroke," Evidence-Based Complementary and Alternative Medicine, vol. 2019, Article ID 1204709, 2019.

[13] L. Zhao, D. Li, H. Zheng et al., "Acupuncture as adjunctive therapy for chronic stable angina," JAMA Internal Medicine, vol. 179, no. 10, p. 1388, 2019.

[14] W. Huang, N. Kutner, and D. L. Bliwise, "Autonomic activation in insomnia: the case for acupuncture," Journal of Clinical Sleep Medicine, vol. 7, no. 1, pp. 95-102, 2011.

[15] S. Schuh-Hofer, R. Wodarski, D. B. Pfau et al., "One night of total sleep deprivation promotes a state of generalized hyperalgesia: a surrogate pain model to study the relationship of insomnia and pain," Pain, vol. 154, no. 9, pp. 1613-1621, 2013.

[16] Y. Huang and Y. Zheng, "Sleep disorder of schizophrenia treated with shallow needling: a randomized controlled trial," Zhongguo Zhen Jiu=Chinese Acupuncture \& Moxibustion, vol. 35, no. 9, pp. 869-873, 2015.

[17] Q. He, Y. F. Yang, and C. L. Wu, "A clinical trial of treatment of primary insomnia of patients with qi-stagnation constitution by shallow acupuncture combined with ear-acupoint pellet-pressing," Zhen Ci Yan Jiu=Acupuncture Research, vol. 44, no. 4, pp. 293-296, 2019.

[18] J. Li, X. Ran, C. Cui, C. Xiang, A. Zhang, and F. Shen, "Instant sedative effect of acupuncture at GV20 on the frequency of electroencephalogram $\alpha$ and $\beta$ waves in a model of sleep deprivation," Experimental and Therapeutic Medicine, vol. 15, no. 6, pp. 5353-5358, 2018.

[19] A. Kalauzi, A. Vuckovic, and T. Bojić, "Topographic distribution of EEG alpha attractor correlation dimension values in wake and drowsy states in humans," International Journal of Psychophysiology, vol. 95, no. 3, pp. 278-291, 2015.

[20] N. Ji, L. Ma, H. Dong, and X. Zhang, "EEG signals feature extraction based on DWT and EMD combined with approximate entropy," Brain Sciences, vol. 9, no. 8, p. 201, 2019.

[21] F. Shayegh, S. Sadri, R. Amirfattahi, and K. Ansari-Asl, "A model-based method for computation of correlation dimension, Lyapunov exponents and synchronization from depth-EEG signals," Computer Methods and Programs in Biomedicine, vol. 113, no. 1, pp. 323-337, 2014.

[22] !!! Invalid citation !!! 22.

[23] U. R. Acharya, O. Faust, N. Kannathal, T. Chua, and S. Laxminarayan, "Non-linear analysis of EEG signals at 
various sleep stages," Comput Methods Programs Biomed, vol. 80, no. 1, pp. 37-45, 2005.

[24] F. Yang and W. Liao, "Approximate entropy: a complexity measure suitable for short data," Zhongguo Yi Liao Qi Xie Za Zhi=Chinese Journal of Medical Instrumentation, vol. 21, no. 5, pp. 283-286, 1997.

[25] I. R. Bell, A. Howerter, N. Jackson, M. Aickin, R. R. Bootzin, and A. J. Brooks, "Nonlinear dynamical systems effects of homeopathic remedies on multiscale entropy and correlation dimension of slow wave sleep EEG in young adults with histories of coffee-induced insomnia," Homeopathy, vol. 101, no. 3, pp. 182-192, 2012.

[26] L. Priano, F. Saccomandi, A. Mauro, and C. Guiot, "Nonlinear recurrence analysis of NREM human sleep microstructure discloses deterministic oscillation patterns related to sleep stage transitions and sleep maintenance," in Proceedings of the 2010 Annual International Conference of the IEEE Engineering in Medicine and Biology, vol. 2010, pp. 49344937, Buenos Aires, Argentina, September 2010.

[27] C. S. O. P. C. M. Association, Chinese Classification and Diagnostic Criteria of Mental Diseases, Vol. 3, Jinan: Shandong Science and Technology Press, Jinan, China, 2001.

[28] A. P. Association, Diagnostic and Statistical Manual of Mental Disorders, American Psychiatric Publishing, Arlington, VA, USA, 5th edition, 2013.

[29] R. Green and W. Shou, "Modeling community population dynamics with the open-source language $R$," Methods in Molecular Biology, vol. 1151, pp. 209-231, 2014.

[30] Y. Li, "Using the open-source statistical language $R$ to analyze the dichotomous Rasch model," Behavior Research Methods, vol. 38, no. 3, pp. 532-541, 2006.

[31] S. M. Pincus, "Approximate entropy as a measure of irregularity for psychiatric serial metrics," Bipolar Disorders, vol. 8, no. 5 Pt 1, pp. 430-440, 2006.

[32] S. M. Pincus, "Approximate entropy as a measure of system complexity," Proceedings of the National Academy of Sciences of the United States of America, vol. 88, no. 6, pp. 2297-2301, 1991.

[33] C. J. Stam, "Nonlinear dynamical analysis of EEG and MEG: review of an emerging field," Clinical Neurophysiology: Official Journal of the International Federation of Clinical Neurophysiology, vol. 116, no. 10, pp. 2266-2301, 2005.

[34] P. Ashwin, "Nonlinear dynamics: synchronization from chaos," Nature, vol. 422, no. 6930, pp. 384-385, 2003.

[35] B. Liu, J. Chen, J. Wang et al., "Altered small-world efficiency of brain functional networks in acupuncture at ST36: a functional MRI study," PLoS One, vol. 7, no. 6, Article ID e39342, 2012.

[36] C. Zhong, L. Bai, R. Dai et al., "Modulatory effects of acupuncture on resting-state networks: a functional MRI study combining independent component analysis and multivariate Granger causality analysis," Journal of Magnetic Resonance Imaging, vol. 35, no. 3, pp. 572-581, 2012.

[37] J. Li, C. Peng, D. Lai et al., "Changes in cerebral glucose metabolism after acupuncture at KI3 in spontaneously hypertensive rats: a positron emission tomography study," Acupuncture in Medicine, vol. 37, no. 2, pp. 107-115, 2019.

[38] B. Libet, "Reflections on the interaction of the mind and brain," Progress in Neurobiology, vol. 78, no. 3-5, pp. 322-326, 2006.

[39] D. Lin, X. Z. Huang, W. Y. Zhuang, Y. Y. Yu, and Q. Wu, "Analysis on insomniac electroencephalogram data after treatment with superficial needling based on approximate entropy and correlation dimensionality," Zhen Ci Yan Jiu= Acupuncture Research, vol. 43, no. 3, pp. 180-184, 2018.

[40] R. Ferri, P. Clochon, F. Bertran et al., "Specific EEG sleep pattern in the prefrontal cortex in primary insomnia," PLos One, vol. 10, no. 1, 2015.

[41] M. A. Colombo, J. R. Ramautar, Y. Wei et al., "Wake highdensity electroencephalographic spatiospectral signatures of insomnia," Sleep, vol. 39, no. 5, pp. 1015-1027, 2016.

[42] D. J. Meyerhoff, A. Mon, T. Metzler, and T. C. Neylan, "Cortical gamma-aminobutyric acid and glutamate in posttraumatic stress disorder and their relationships to self-reported sleep quality," Sleep, vol. 37, no. 5, pp. 893-900, 2014.

[43] N. R. Garge, G. P. Page, A. P. Sprague, B. S. Gorman, and D. B. Allison, "Reproducible clusters from microarray research: whither?," BMC Bioinformatics, vol. 6, no. Suppl 2, p. S10, 2005.

[44] W. Guo, Q. Zhou, Y. Jia, and J. Xu, "Cluster and factor analysis of elements in serum and urine of diabetic patients with peripheral neuropathy and healthy people," Biological Trace Element Research, vol. 194, no. 1, pp. 48-57, 2020.

[45] X. Hu, Z. Liu, W. Chen et al., "Individual differences in the accuracy of judgments of learning are related to the gray matter volume and functional connectivity of the left midinsula," Frontiers in Human Neuroscience, vol. 11, p. 399, 2017.

[46] K. J. Stam, D. N. L. J. Tavy, B. Jelles, H. A. M. Achtereekte, J. P. J. Slaets, and R. W. M. Keunen, "Non-linear dynamical analysis of multichannel EEG: clinical applications in dementia and Parkinson's disease," Brain Topography, vol. 7, no. 2, pp. 141-150, 1994.

[47] T. Kobayashi, K. Misaki, H. Nakagawa et al., "Correlation dimension of the human sleep electroencephalogram," Psychiatry and Clinical Neurosciences, vol. 54, no. 1, pp. 11-16, 2000.

[48] F. Zhou, Y. Zhao, M. Huang, X. Zeng, B. Wang, and H. Gong, "Disrupted interhemispheric functional connectivity in chronic insomnia disorder: a resting-state fMRI study," Neuropsychiatric Disease and Treatment, vol. 14, pp. 12291240, 2018. 